\title{
Clinical Trial of Bleaching Treatment With $10 \%$ All-Trans Retinol Gel
}

\author{
Kotaro Yoshimura, MD, ${ }^{*}$ Akira Momosawa, MD, ${ }^{*}$ Emiko Aiba, MD, * Katsujiro Sato, \\ MD, Daisuke Matsumoto, MD, ${ }^{*}$ Yoko Mitoma, MD, ${ }^{*}$ KiYonori Harit, MD, \\ Takao Aoyama, PhD, ${ }^{\dagger}$ And Tatsuji IGa, PhD ${ }^{\dagger}$ \\ *Department of Plastic and Reconstructive Surgery, and Department of Pharmacy, University of Tokyo, Graduate \\ School of Medicine, Tokyo, Japan
}

BACKGROUND. Although an aggressive use of tretinoin along with hydroquinone enables an efficient treatment of hyperpigmented skin lesions, irritant dermatitis remains to be solved. OBJECTIVE. To evaluate the efficiency and adverse effects of $10 \%$ all-trans retinol (ROL) gel for improvement of skin hyperpigmentation.

METHODS. Ten-percent ROL gel was used instead of $0.1 \%$ tretinoin gel in our two-phased bleaching protocol (bleaching and healing phases); $5 \%$ hydroquinone and $7 \%$ lactic acid ointment were used along with ROL gel in the bleaching phase ( 2 to 6 weeks). Five-percent hydroquinone and $7 \%$ ascorbic acid ointment were used alone during the healing phase (4 to 6 weeks). Twenty-one Japanese patients with hyperpigmented lesions on the face were enrolled in this study, and 18 patients who were followed for more than 10 weeks were analyzed. RESULTS. Improvement of pigmentation was seen in 16 of 18 patients after an average treatment period of 11.3 weeks, and in 6 patients, pigmentation was almost eliminated after treatment. Erythema and scaling were seen, however, during the bleaching phase as well as the bleaching treatment with tretinoin gel.

CONCLUSION. ROL can improve skin hyperpigmentation to a similar extent to tretinoin when used at high concentration, whereas it induces irritant dermatitis as well.

K. YOSHIMURA, MD, A. MOMOSAWA, MD, E. AIBA, MD, K. SATO, MD, D. MATSUMOTO, MD, Y. MITOMA, MD, K. HARII, MD, T. AOYAMA, PhD, AND T. IGA, PhD HAVE INDICATED NO SIGNIFICANT INTEREST WITH COMMERCIAL SUPPORTERS.

TRETINOIN AND hydroquinone have been used successfully for treatment of skin hyperpigmentation since Kligman and Willis ${ }^{1}$ introduced their bleaching formula. Several modified protocols have been reported, ${ }^{2}$ and the authors also proposed an aggressive bleaching therapy, in which tretinoin and hydroquinone were used separately and corticosteroids were not used. ${ }^{3-6}$ In our facility, more than 8,500 cases with various kinds of hyperpigmented lesions have been successfully treated with our protocol and its modifications since 1995. Virtually the only adverse side effect of the strong bleaching treatment is irritant dermatitis. Further investigations and clinical trials would be worthwhile in order to reduce the displeasing side effects without losing bleaching ability.

All-trans retinol (ROL) and all-trans retinal (RAL) have been considered to be less irritating than tretinoin if applied at relatively low $(0.1 \%$ to $1.6 \%)$ concentra-

Address correspondence and requests for reprints to: Kotaro Yoshimura, MD, Department of Plastic and Reconstructive Surgery, Graduate School of Medicine, University of Tokyo, 7-3-1, Hongo, Bunkyo-Ku, Tokyo 113-8655, Japan, or e-mail: yoshimura-pla@h.u-tokyo.ac.jp. tion. ${ }^{7,8}$ However, because binding affinity to specific nuclear receptors (retinoic acid receptors; RARs) of ROL or RAL is far less than that of tretinoin, beneficial effects are also expected to be very moderate compared with tretinoin. Our previous study revealed that heparin-binding epidermal growth factor-like growth factor (HB-EGF) mRNA, which plays a critical role in epidermal growth after retinoid treatment, ${ }^{9,10}$ was upregulated by ROL or RAL to a similar extent as tretinoin, but only when ROL or RAL was used at 40 to 100 times higher concentration than tretinoin. ${ }^{11}$ Because proliferation of keratinocytes and acceleration of epidermal turnover appear to be the two main mechanisms of accelerated melanin discharge by retinoids, ${ }^{11,12}$ we consider that promotion of $\mathrm{HB}$ EGF mRNA expression may be used as an important index to evaluate the depigmenting ability of topical retinoids.

Based on the data mentioned previously here, we prepared $10 \%$ ROL aqueous gel because ROL gel of 100 times higher concentration than tretinoin can be expected to show similar clinical effects to tretinoin gel $(0.1 \%)$. In this trial study, the ROL gel was clinically 
used for depigmenting in order to estimate its bleaching potential and the extent of adverse side effects.

\section{Methods}

\section{Preparation of Ointments}

ROL aqueous gel $(10 \%)$ was originally prepared and packed into small stainless tubes $(5 \mathrm{~g}$ each) at the Department of Pharmacy, University of Tokyo Graduate School of Medicine. The precise regimen of ROL aqueous gel for $100 \mathrm{~g}$ was as follows: all-trans ROL 10 g (Sigma Chemical, St. Louis, MO), Carbopol $9401 \mathrm{~g}$ (Goodrich Chemical, Hounlow, UK), polyoxyethylene oleyl ether $2.5 \mathrm{~g}$ (Kao, Tokyo, Japan), methyl phydroxybenzoate $0.026 \mathrm{~g}$ (Wako Pure Chemical Industries, Osaka, Japan), propyl p-hydroxybenzoate $0.014 \mathrm{~g}$ (Wako Pure Chemical Industries), 10\% sodium hydroxide aqueous solution $0.6 \mathrm{~mL}$, and purified water 86 g. An ointment including 5\% hydroquinone and 7\% lactic acid (HQ-LA ointment) and an ointment including 5\% hydroquinone and 7\% ascorbic acid (HQ-AA ointment) were also prepared. Plastibase (petrolatum polyethylene ointment base; Taisho Pharmacology, Osaka, Japan) was used as the ointment base of the HQ-LA ointment, whereas the hydrophilic ointment was used for the HQ-AA ointments. Because ROL gel, HQ-LA, and HQ-AA ointments (especially ROL gel) are pharmacologically unstable, fresh ointments were prepared at least once a month and stored in a dark and cool $\left(4^{\circ} \mathrm{C}\right)$ place.

\section{Evaluations of Results}

Photographs were taken for every patient at baseline and after treatment with a high-resolution digital camera (Canon EOS-D30). The percentage of pigmentary clearance was evaluated via the photographs by two experienced plastic surgeons who did not perform this treatment. The mean data of the pigmentary clearance of each patient were classified into four categories: excellent $(90 \%$ or more clearance), good (60\% to less than $90 \%$ clearance), fair $(30 \%$ to less than $60 \%$ clearance), and poor (less than $30 \%$ clearance).

\section{Patients}

Each ointment was topically applied under signed informed consent in 21 Japanese women with hyperpigmented skin lesions on the face, and 18 of them followed for more than 10 weeks were analyzed in this study. The other three patients stopped treatment because of irritant dermatitis induced by ROL gel and/ or hydroquinone. The age of patients ranged from 20 to 67 years old (age, 43.2 \pm 7.0 ; mean $\pm S D$ ). Thirteen patients had solar lentigines. Five had melasma, and four had ephelides. Four patients had two kinds of pigmented lesions.

\section{Treatment Protocol}

Our bleaching protocol is composed of two phases, a bleaching phase and a healing phase. In the bleaching phase, the pigmentation is aggressively treated, and transient adverse skin effects such as erythema and irritation are usually observed. Once satisfactory improvement is obtained, the healing phase is started in order to reduce the erythema and inflammation, taking care not to induce new postinflammatory hyperpigmentation.

1. Bleaching phase: $10 \%$ ROL gel and HQ-LA ointment were applied twice a day. ROL gel was carefully applied only on pigmented areas using a small cotton-tip applicator, and subsequently, HQ-LA ointment was widely applied with the fingers beyond the pigmented area (e.g., all over the face). Patients were requested to visit our hospital at 1,2, 4, 6, and 8 weeks after starting this treatment and every 2 weeks thereafter. In most cases, it took 2 to 6 weeks to finish this phase.

2. Healing phase: After sufficient improvement of pigmentation was obtained, the application of ROL gel and HQ-LA ointment was discontinued, and application of HQ-AA ointment all over the face was started. HQ-AA ointment was used until the erythema was almost eliminated, and it took 4 to 6 weeks to complete this phase. Topical corticosteroids were not employed either in the bleaching or healing phase.

\section{Results}

The average treatment period of 18 patients was 11.3 weeks and ranged from 8 to 16 weeks. Erythema and scaling appeared during the first week in most cases. Compared with thousands of our cases treated with $0.1 \%$ tretinoin gel (our preparation regimen is available in Yoshimura et $\mathrm{al}^{4}{ }^{4}$ ), we thought that $10 \% \mathrm{ROL}$ gel induced a slightly less degree of erythema and a higher degree of scaling in the first 2 weeks, although statistical analysis was not performed. In addition, tolerance to the active reagent seemed to be acquired earlier in the ROL treatment than with tretinoin. In patients who achieved tolerance as early as 2 weeks, further improvement was deemed unlikely, and thus, the results were categorized as poor and fair. 
Table 1. Summarized Data of Objective Evaluations

\begin{tabular}{ll}
\hline Excellent & $6(33.3 \%)$ \\
Good & $7(38.9 \%)$ \\
Fair & $3(16.7 \%)$ \\
Poor & $2(11.1 \%)$ \\
Total & $18(100 \%)$ \\
\hline
\end{tabular}

The clinical results were summarized in Table 1 . Six patients $(33.3 \%)$ were evaluated as "excellent," seven cases $(38.9 \%)$ as "good," three cases $(16.7 \%)$ as "fair," and two cases $(11.1 \%)$ as "poor." Some improvement was seen in 16 of 18 patients $(88.9 \%)$. Most of fair and poor cases had very mild or no skin reactions such as scaling and erythema during the bleaching phase. The representative four cases are shown in Figures 1-4.

\section{Discussion}

In bleaching treatment with tretinoin and hydroquinone, we believe that the role of tretinoin is to wash melanin granules out of epidermis, whereas that of hydroquinone is to suppress strongly new melanin production. ${ }^{11,12}$ Tretinoin induces exfoliation, and thus, it appears to also act as a penetration enhancer of hydroquinone. However, tretinoin has a specific effect as a discharger of epidermal melanin that cannot be performed by other penetration-enhancing procedures such as alpha hydroxyacid peeling and microdermabrasion. These exfoliating procedures simply induce the subsequent normal wound healing process and discharge epidermal melanin only very weakly. On the other hand, it is suggested that tretinoin promotes discharge of melanin granules in epidermis by (1) accelerating epidermal turnover (differentiation of keratinocytes) in a direct manner and also (2) promoting epidermal growth (proliferation of keratinocytes) in an indirect manner; the latter of the two effects was found to be mediated by HB-EGF secreted by suprabasal keratinocytes. ${ }^{9,10,13}$ Indeed, the successful results of an aggressive bleaching treatment with tretinoin and hydroquinone cannot be obtained by combination therapies of other exfoliating procedures and topical hydroquinone. ${ }^{4}$ These observations suggest a specific ability of retinoids as a melanin discharger.

As mentioned previously here, the mechanism of this bleaching therapy seems to be very simple: Tretinoin accelerates the loss of epidermis melanin, whereas hydroquinone suppresses the production. The bleaching therapy with tretinoin appears to be only effective for epidermal pigmentation because we
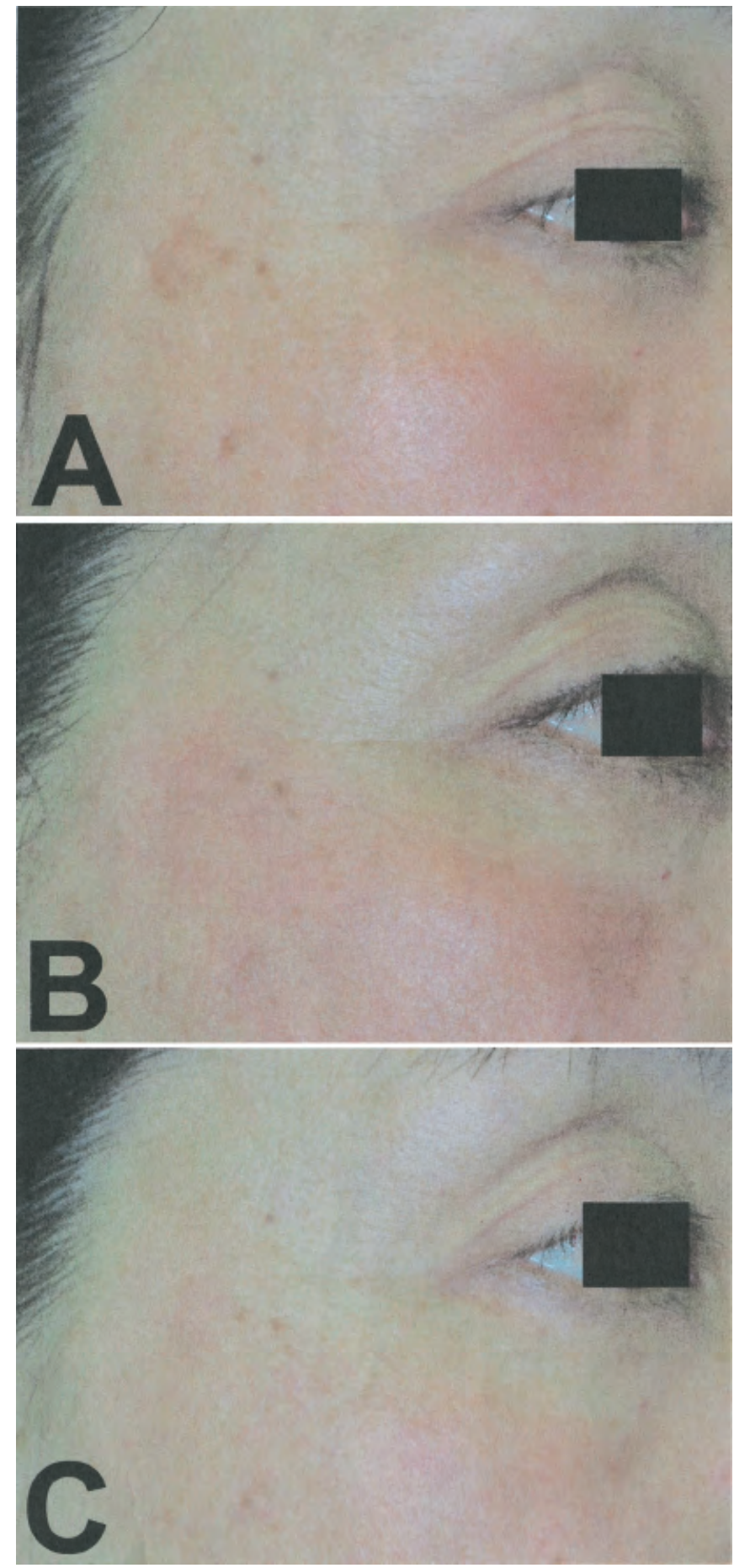

Figure 1. Case 1. A 46-year-old woman with a solar lentigine and melasma on her cheek underwent the treatment ( $A$, before treatment); $10 \%$ ROL gel was used for 4 weeks together with HQLA ointment ( $B$, at 4 weeks; after bleaching treatment), followed by application of HQ-AA ointment alone for 4 weeks. Both the solar lentigine and melasma almost disappeared after 8 weeks of treatment (C, at 8 weeks).

confirmed histologically that accumulated melanin granules around the basal layer were cleared up after treatment with tretinoin and hydroquinone, but the 


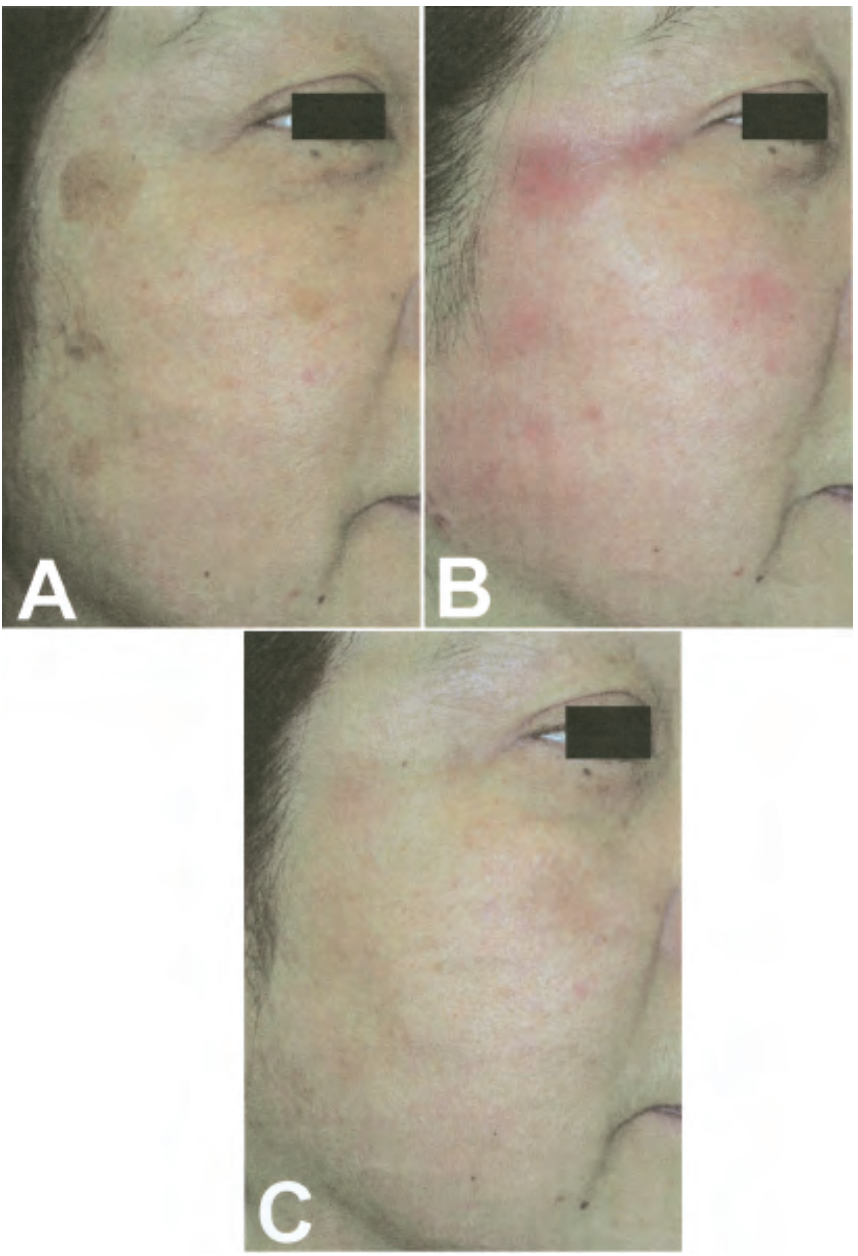

Figure 2. Case 2. A 54-year-old woman with several solar lentigines on her right cheek underwent the treatment ( $\mathrm{A}$, before treatment); $10 \%$ ROL gel was used for 2 weeks together with HQ-LA ointment, followed by application of HQ-AA ointment alone for 6 weeks. Significant erythema and irritation on the treated area were observed 1-3 weeks after starting the treatment ( $B$, at 2 weeks; after bleaching phase). The erythema almost disappeared after 6-week healing phase, although slight postinflammatory hyperpigmentation was left on the treated areas (C, at 8 weeks).

melanin deposits in the dermis appeared not to change in acquired bilateral nevus of Ota-like macules, which has both epidermal and dermal pigmentation (in preparation).

The authors recently reported that ROL and RAL can markedly induce HB-EGF mRNA upregulation at a pharmacologic concentration $(0.01$ to $1 \mathrm{mmol} / \mathrm{L})$ in cultured human keratinocytes. ${ }^{11}$ However, in cases of ROL and RAL, 40 to 100 times higher concentration was required in order to upregulate HB-EGF mRNA to a similar extent to tretinoin. ${ }^{11}$ In this study, we prepared and used $10 \%$ ROL gel, which is 100 times higher concentration that $0.1 \%$ tretinoin gel because it could be expected to clinically induce HB-EGF expression in the epidermis as well as topical $0.1 \%$
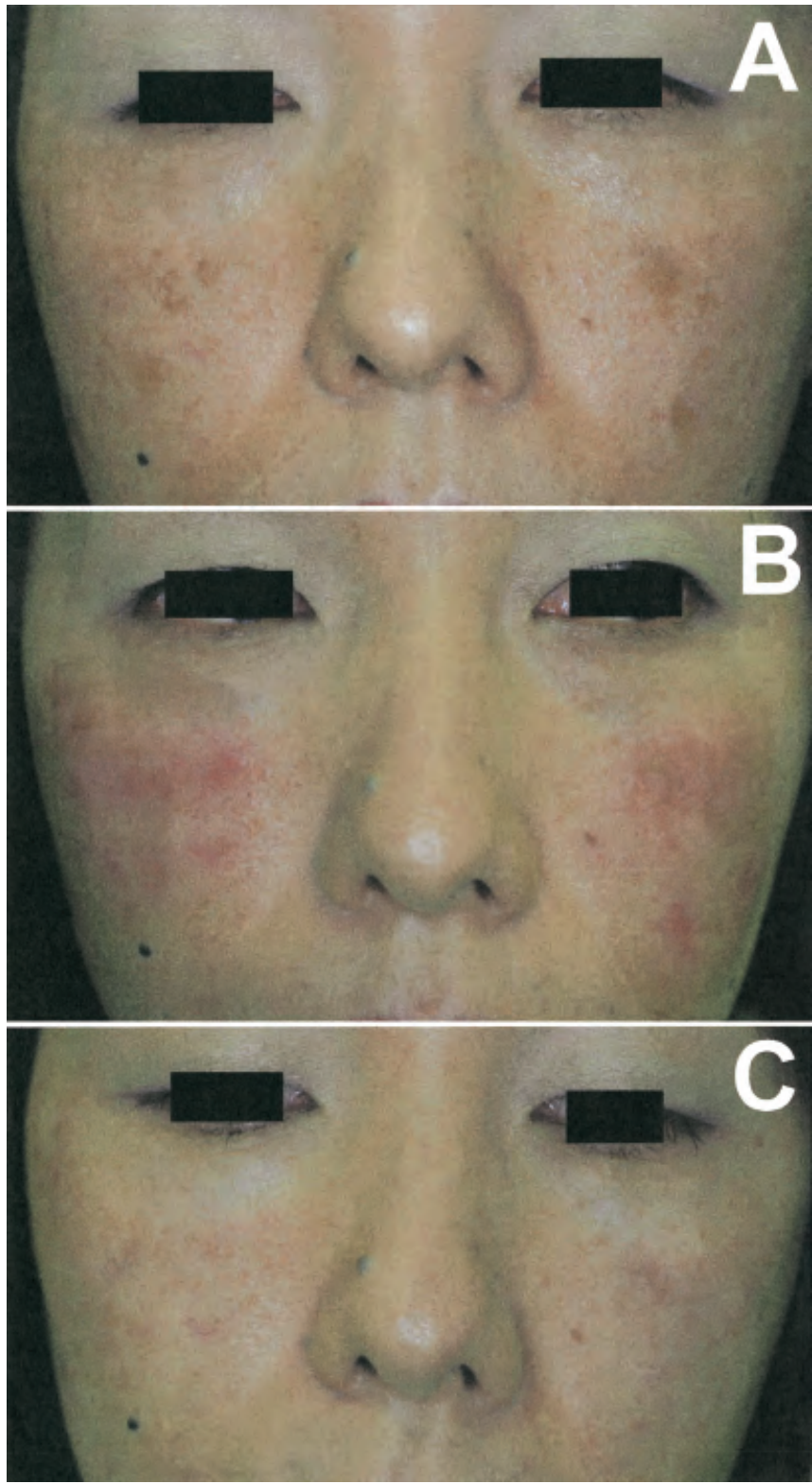

Figure 3. Case 3. A 32-year-old woman with melasma and some solar lentigines underwent the treatment ( $\mathrm{A}$, before treatment); $10 \% \mathrm{ROL}$ gel was used for 4 weeks combined with HQ-LA ointment, followed by single application of HQ-AA ointment for 4 weeks. Irritant dermatitis was seen on the treated areas throughout the bleaching phase, but pigmentation was improved significantly by day 28 (B, at 4 weeks; after bleaching phase). After 4 weeks of healing phase, erythema disappeared without leaving postinflammatory hyperpigmentation (C, at 8 weeks).

tretinoin. The results suggest that $10 \%$ ROL gel was comparable in effectiveness to $0.1 \%$ tretinoin gel in improving skin hyperpigmentation compared with our bleaching experience with thousands of patients treated with $0.1 \%$ tretinoin gel.

ROL and RAL are considered to work predominantly after conversion to tretinoin. The binding affinities of ROL and RAL to RARs are quite low ${ }^{14}$ 


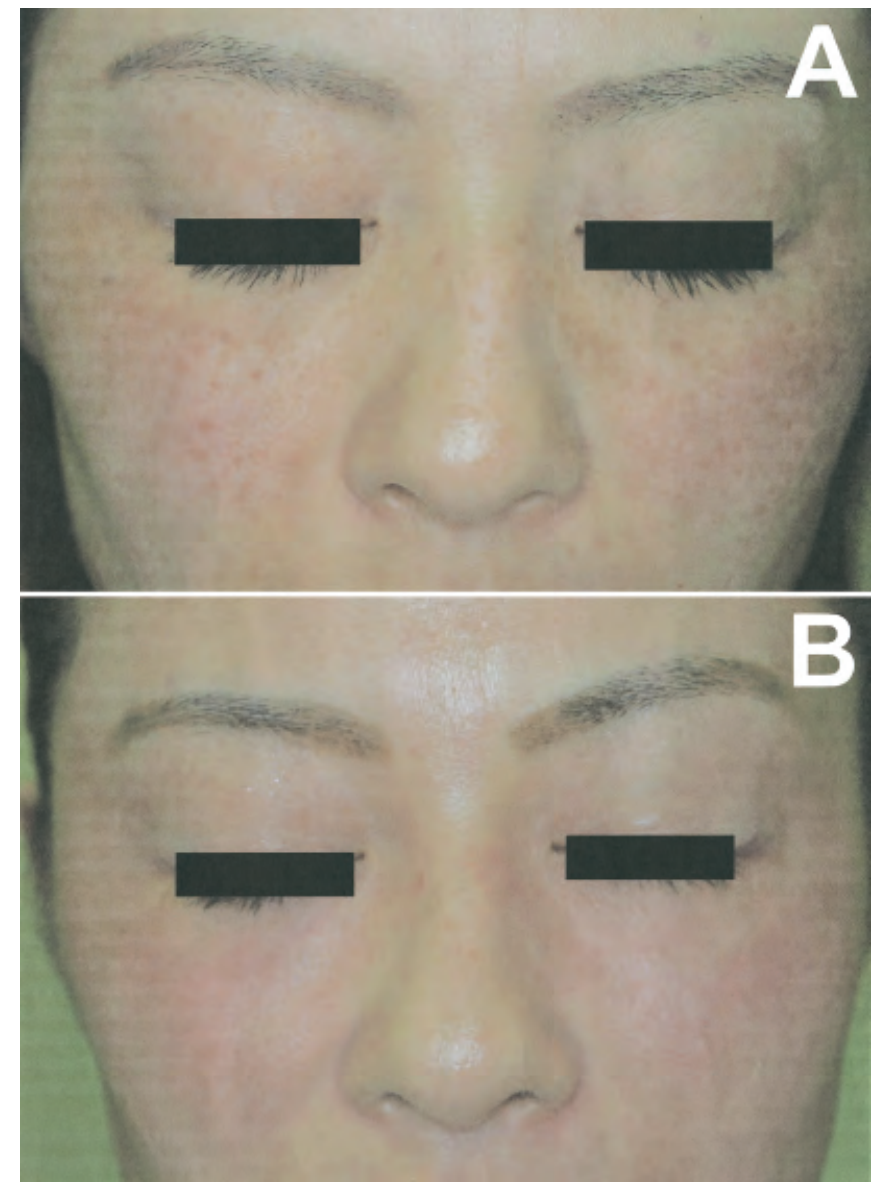

Figure 4. Case 4. A 34-year-old woman with ephelides underwent the treatment ( $A$, before treatment). Bleaching was performed with $10 \%$ ROL gel and HQ-LA ointment for 4 weeks; thereafter, HQ-AA ointment alone was used for 6 weeks. The pigmented spots were almost eliminated, and mild rosy glow was still seen at 10 weeks (B, at 10 weeks).

so that their biologic activity should result from their oxidative transformation into tretinoin by epidermal keratinocytes. This conversion to tretinoin after topical application of ROL and RAL was revealed to occur in a study using skin organ culture ${ }^{15}$ although the conversion rate has not been clearly measured so far. However, there have been some reports suggesting the existence of other pathways than that mediated by nuclear receptors. ${ }^{16}$ Thus, ROL seems to show its biologic activity through direct binding to RARs, transformation into tretinoin, and other pathways. HB-EGF mRNA upregulation in suprabasal keratinocytes can be expected to occur in vivo after topical application of ROL.

ROL is known to show far less severe side effects (such as irritation) than tretinoin, at least when used at similar concentration, and thus has been thought to be more tolerable and may be of great value in clinical use. However, this study revealed that even ROL can induce irritant contact dermatitis to a similar extent as tretinoin when used at a 100-times higher concentration, although it also showed beneficial effects similar to tretinoin. Retinyl esters such as retinyl palmitate and retinyl acetate are pharmacologically very stable, and topical formulations of them are widely available mainly as cosmetics. Although retinyl esters are known to show even less irritation than ROL, their biologic activity as retinoids appears to be far less than that of pure $\mathrm{ROL}$ or $\mathrm{RAL}^{17}$ and cannot be expected to be effectively transformed to retinoic acids and to work as drugs.

It remains unknown what percentages of ROL in the prepared ROL gel were absorbed into the skin. Unlike the $0.1 \%$ tretinoin gel we used in which more than $96 \%$ of the gel is composed of water, the $10 \%$ ROL gel contains $10 \%$ ROL, whereas water formed only $86 \%$ of it. The permeability of ROL in the $10 \%$ ROL gel may be much less than that of tretinoin in the $0.1 \%$ tretinoin gel. Therefore, $5 \%$ ROL gel, which should contain more water, may show similar depigmenting effects to the $10 \%$ ROL gel.

In this study, we showed that ROL can play a depigmenting role as well as tretinoin. However, the cost performance of the ROL gel is very poor compared with the tretinoin gel, as far as our preparations are concerned. Purified ROL is commercially available, but $1 \mathrm{~g}$ costs almost the same as $1 \mathrm{~g}$ of tretinoin. Because 100 times the amount of the working ingredient was required to prepare the ROL gel, it costs 100 times more. In light of this, it seems to be impractical to use clinically the $10 \%$ ROL gel that we prepared.

\section{Conclusions}

This clinical trial revealed that $10 \%$ ROL gel clinically show a considerable bleaching effect, which seems to be as good as that of $0.1 \%$ tretinoin gel, but the ROL gel also induced adverse side effects, such as irritant dermatitis, to a similar degree as tretinoin gel.

\section{References}

1. Kligman AM, Willis I. A new formula for depigmenting human skin. Arch Dermatol 1975;111:40-8.

2. Gano SE, Garcia RL. Topical tretinoin, hydroquinone, and betamethasone valerate in the therapy of melasma. Cutis 1979;23:239-41.

3. Yoshimura K, Harii K, Shibuya F, et al A new bleaching protocol for hyperpigmented skin lesions with a high concentration of alltrans retinoic acid aqueous gel. Aesthetic Plast Surg 1999;23:285-91.

4. Yoshimura K, Harii K, Aoyama T, Iga T. Experience of a strong bleaching treatment for skin hyperpigmentation in Orientals. Plast Reconstr Surg 2000;105:1097-108.

5. Yoshimura K, Harii K, Masuda Y, et al. Usefulness of a narrowband reflectance spectrophotometer in evaluating effects of depigmenting treatment. Aesthetic Plast Surg 2001;25:129-33. 
6. Yoshimura K, Momosawa A, Watanabe A, et al. Color improvement of the nipple-areola complex by optimal use of tretinoin and hydroquinone. Dermatol Surg, in press.

7. Kang S, Duell EA, Fisher GJ, et al. Application of retinol to human skin in vivo induces epidermal hyperplasia and cellular retinoid binding proteins characteristic of retinoic acid but without measurable retinoic acid levels or irritation. J Invest Dermatol 1995; 105:549-56.

8. Fluhr JW, Vienne MP, Lauze C, et al Tolerance profile of retinol, retinaldehyde and retinoic acid under maximized and long-term clinical conditions. Dermatology 1999;199(Suppl 1):57-60.

9. Xiao JH, Feng X, Di W, et al Identification of heparin-binding EGFlike growth factor as a target in intercellular regulation of epidermal basal cell growth by suprabasal retinoic acid receptors. EMBO J 1999;18:1539-48.

10. Varani J, Zeigler M, Dame MK, et al. Heparin-binding epidermalgrowth-factor-like growth factor activation of keratinocytes ErbB receptors mediates epidermal hyperplasia, a prominent side-effect of retinoid therapy. J Invest Dermatol 2001;117:1335-41.

11. Yoshimura K, Uchida G, Okazaki M, et al. Differential expression of heparin-binding EGF-like growth factor (HB-EGF) mRNA in normal human keratinocytes induced by a variety of natural and synthetic retinoids. Exp Dermatol, in press.

\section{Commentary}

Retinoid research began the entire cosmeceutical era when it was proven that tretinoin could actually regenerate collagen, improve dyspigmentation, and enhance skin texture. This body of research that began in the 1960s is continuing as retinoid technology is being refined in the pharmaceutical market and adapted to the over-the-counter market. This article deals with the use of an extemporaneously compounded $10 \%$ all-trans ROL gel. Retinoids are basically yellow, fat-soluble, lipophilic compounds that are sensitive to light, oxygen, and heat. They chemically conform to an aromatic ring structure with a carbon chain and terminal group that can be varied to determine the function, stability, and irritation profile of a retinoid on the skin. It is research into this terminal group that is the current focus of retinoid research. ROL, the active used in this study, is a naturally occurring form of vitamin $\mathrm{A}$, whereas retinoic acid (tretinoin) and retinaldehyde are vitamin A metabolites. Retinyl acetate, retinyl propionate, and retinyl palmitate are vitamin A esters currently used in the over-the-counter market. These various retinoids have different formulations issues and different skin effects. Retinyl esters are popular in cosmetics, as they are less sensitive to oxidation, thus demonstrating greater stability and a longer shelf life, and are the least irritating to the skin. On the other hand, retinoic acid is relegated to the prescription market due to its current classification as a drug and its high skin irritation profile. ROL, the subject of this article, is less irritating than retinoic acid but is highly unstable. The most interesting aspect of these vitamin A metabolites is the theoretical interconversion of one
12. Yoshimura K, Tsukamoto K, Okazaki M, et al Effects of all-trans retinoic acid on melanogenesis in pigmented skin equivalents and monolayer culture of melanocytes. J Dermatol Sci 2001;27(Suppl 1):68-75.

13. Stoll SW, Elder JT. Retinoid regulation of heparin-binding EGF-like growth factor gene expression in human keratinocytes and skin. Exp Dermatol 1998;7:391-7.

14. Crettaz M, Baron A, Siegenthaler G, Hunziker W. Ligand specificities of recombinant retinoic acid receptors RA alpha and RAR beta. Biochem J 1990;272:391-7.

15. Bailly J, Crettaz M, Schifflers MH, Marty JP. In vitro metabolism by human skin and fibroblasts of retinol, retinal and retinoic acid. Exp Dermatol 1998;7:27-34.

16. Saurat JH, Sorg O, Didierjean L. New concepts for delivery of topical retinoid activity to human skin. In: Nau H, Blaner WS, eds. Retinoids. Berlin: Springer, 1999:521-38.

17. Kligman AM. The growing importance of topical retinoids in clinical dermatology: a retrospective and prospective analysis. J Am Acad Dermatol 1998;39:S2-7.

to another. For example, the retinyl esters can be converted to ROL via hydrolysis. ROL can be converted to retinaldehyde via oxidation, and another oxidation step can convert retinaldehyde to retinoic acid. This is particularly important when one realizes that the enzymatic machinery to perform the conversions resides within the skin. Current research is aimed at determining how much conversion can realistically occur. It is unknown how much ROL can be converted to retinoic acid over what period of time. The interest has been in quantitating how much ROL, an over-the-counter vitamin, is converted to retinoic acid, a prescription product. The chemistry becomes quite interesting because it blurs the current distinction between a vitamin, a cosmetic active, and a prescription medication. This article investigates the effect of ROL in lightening skin, a common worldwide problem especially in women of color. ROL is present in some over-the-counter skin lightening and cosmeceutical preparations usually in concentrations of $2 \%$ or less, because of tremendous irritation induced by this active. This research that uses $10 \%$ all-trans ROL gel is particularly notable for the irritation sustained by the patients. Combining the ROL with lactic acid, a penetration enhancer, furthers the penetration of ROL into the skin, but also enhances the irritation. These researchers had to prepare the study ROL formulation monthly because of instability. Thus, ROL products may eventually augment those hydroquinonebased skin lightening products currently used in the United States, once the irritation and stability issues are resolved.

$$
\text { Zoe Diana Draelos, MD }
$$
High Point, NC 